\title{
Coronary artery calcium testing
}

In the PROMISE trial, presented at the ESC Congress 2017, coronary artery calcium (CAC) had a high sensitivity for future cardiovascular events in patients with stable chest pain, whereas functional testing had high specificity for predicting events. Given the similarly modest discriminatory capabilities of these tests, a combined approach might be appropriate.

Investigators of the prospective, multicentre, randomized PROMISE trial sought to compare the prognostic value of CAC testing with functional stress testing in stable outpatients presenting with suspected coronary artery disease (CAD). Patients presenting with stable chest pain were randomly assigned to functional testing or anatomical testing (most of whom underwent CAC testing). CAC and functional test results were stratified as normal, mildly normal, moderately abnormal, or severely abnormal. The primary end point was all-cause death, myocardial infarction, or hospitalization for unstable angina.

In total, 4,209 patients who received CAC testing and 4,602 who received functional testing were followed up for a median of 26.1 months. Event rates were not different between the two diagnostic groups. An abnormal functional test result was more specific for predicting events compared with CAC (78.6\% versus $35.2 \%$; $P<0.001)$. However, $\mathrm{CAC}$ testing was more sensitive than functional testing in predicting events; a positive CAC test (score $>0$ ) identified $84 \%$ of total events, whereas mild, moderate, or severely abnormal functional test results identified only $43 \%$ of total death, myocardial infarction, and unstable angina events. Overall discriminatory capacity for predicting the primary end point was not different between CAC and functional testing.

"The discriminatory ability of CAC suggests that it may have a role in the initial evaluation of new onset stable chest pain," conclude the investigators. "However, both approaches have strengths to detect future cardiovascular events in patients with stable $\mathrm{CAD}$, and a combined tiered approach may be most prudent."

Karina Huynh

ORIGINAL ARTICLE Budoff, M. J. et al. The prognostic value of coronary artery calcium in the PROMISE study. Circulation http://dx.doi.org/10.1161/CIRCULATIONAHA.117.030578(2017) 